\title{
The $n$ me gene family in zebrafish oogenesis and early development
}

\author{
T. Desvignes ${ }^{a, b}, C$. Fauvel $^{b}$ and J. Bobe ${ }^{a, *}$ \\ a INRA, UR1037 SCRIBE, Campus de Beaulieu, 35042 Rennes, France \\ ${ }^{\mathrm{b}}$ IFREMER, LALR, 34250 Palavas-Les-Flots, France \\ *: Corresponding author : Julien Bobe, email address : Julien.Bobe@rennes.inra.fr
}

\begin{abstract}
:
After the recent report of the expression of several nme genes in the zebrafish gonads, the present study aimed at further analyzing the expression of $n m e$ genes in the ovary with special attention for the nme transcripts that are maternally inherited and could thus participate in the determination of oocyte developmental competence. The expression levels of all groups I and II nme genes were characterized by QPCR in a panel of zebrafish tissues. The nme genes exhibiting an ovarian expression were subsequently monitored throughout oogenesis and early development, and their expression sites characterized using in situ hybridization. Here, we show that nme2b1, nme3, nme4, and nme6 are highly expressed in the ovary and present in the zebrafish oocyte throughout oogenesis. While the four transcripts are maternally inherited, nme 3 and nme 6 display a typical maternal profile and are detected in the zebrafish early embryo. In contrast to nme3, nme6, abundance exhibits a sharp decrease during early embryogenesis. After zygotic genome activation, we observed an increased expression of nme2b1, nme2b2, nme3, and nme6. The present study provides a comprehensive overview of the expression of nme family members during zebrafish oogenesis and early development. In addition, the maternal origin of two nme transcripts in the early embryo is reported here for the first time in any vertebrate species. Together, our observations suggest an important role of the nme family in oocyte and embryo development in vertebrates.
\end{abstract}

Keywords : NDPK - Nm23 - Oocyte - Maternal - Teleost - Vertebrate 


\section{Abbreviations}

Hpf: Hour post-fertilization

BSA: Bovine Serum Albumin

MZT: Maternal-to-zygotic transition

MBT: Mid-Blastula Transition

\section{Introduction}

The Nme gene family is involved in multiple physiological and pathological processes such as cellular differentiation, development, metastatic dissemination, and cilia functions (Boissan et al., 2009 ; Biggs et al., 1990). Nme proteins can be separated in 2 different groups - group I and group II - based on their nucleoside diphosphate kinase (NDPK) activity and evolutionary history (Boissan et al., 2009 ; Desvignes et al., 2010). While genes of the group II did not undergo major evolutionary events in vertebrates, genes of the group I underwent several independent duplications in the vertebrate lineage. For instance, mammalian Nme1, Nme2, Nme3, and Nme4 genes are co-orthologs of Awd, the only group I Nme gene found in the Drosophila genome (Desvignes et al., 2010). Similarly, both mammalian Nme1 and Nme2 genes are co-orthologs of zebrafish nme2a, $n m e 2 b 1$, and $n m e 2 b 2$ genes (Desvignes et al., 2009). Despite the known importance of Nme genes in several human pathologies and their use as clinical markers of tumor aggressiveness, their role in non-cancerous physiological processes has, in contrast, received far less attention. The major role of the group I Nme gene awd during Drosophila oogenesis and development has been thoroughly documented (Timmons and Shearn, 2000 ; Woolworth et al., 2009). In contrast, the role of Nme proteins in oogenesis and development has been poorly documented in vertebrates. However, the expression of specific nme genes during development has been reported in several mammalian (Lakso et al., 1992 ; Amrein et al., 2005 ; Carotenuto et al., 2006 ; Postel et al., 2009) and nonmammalian species (Ouatas et al., 1998 ; Murphy et al., 2000 ; Bilitou et al., 2009). More recently, we reported an intriguingly high expression of several nme genes in zebrafish gonads, and more specifically in the ovary (Desvignes et al., 2009). In vertebrates, early development relies on maternal gene products stored into the egg during oogenesis as the genome of the embryo does not start to produce its own RNAs until the MZT (Maternal-to-Zygotic Transition) (see Tadros and Lipshitz, 2009 for review). In mammals, MZT occurs after a few embryonic divisions, while it occurs during Mid-Blastula Transition (MBT) in teleost fish and amphibians. In zebrafish, MBT occurs around 3 to 4 hours postfertilization (Kane and Kimmel, 1993) but is more progressive than initially thought (Mathavan et al., 2005). Before this transition, all developmental processes are driven and supported by maternal gene products, RNA and proteins, that are stored into the oocyte during oogenesis (Pelegri, 2003 ; Dosch et al., 2004 ; Abrams and Mullins, 2009). Post-MBT development is also, but to a lesser extent, still dependent on maternally-inherited gene products, (Wagner et al., 2004). In this context, fertilization and developmental success of zebrafish embryos are dependent on the storage and processing of RNA and proteins that occurs in the oocyte prior to fertilization throughout oogenesis. The dynamic of gene expression in the oocyte during oogenesis is thus important to study in order 
to gain insight into the molecular mechanisms that determine oocyte developmental competence (i.e. oocyte quality) (Bobe and Labbe, 2010).

After the recent report of the expression of several nme genes in the zebrafish gonads, the present study aimed at further analyzing the expression of nme genes in the ovary with special interest for the nme transcripts that are maternallyinherited and could thus participate in the determination of oocyte developmental competence. The present study also aimed at characterizing the dynamic profiles of maternally-inherited nme transcripts in the early embryo, prior to the activation of the embryonic genome. In order to achieve these goals, the expression levels of all group I and group II $n m e$ genes were characterized in a panel of zebrafish tissues. Nme genes exhibiting a significant ovarian expression were subsequently monitored throughout oogenesis and their expression sites characterized using in situ hybridization. For the $n m e$ transcripts exhibiting a maternal profile, transcript abundance was subsequently monitored during early embryonic development.

\section{Materials and Methods}

\section{Animals and sampling}

Investigations were conducted according to the guiding principles for the use and care of laboratory animals and in compliance with French and European regulations on animal welfare (DDSV approval \#35-31). All zebrafish Danio rerio were obtained from the fish rearing facility at INRA-SCRIBE (Rennes, France). For all tissue collections, male or female zebrafish were anesthetized and subsequently killed by head sectioning. Tissues were then sampled, snap-frozen in liquid nitrogen and stored at $-80^{\circ} \mathrm{C}$ until RNA extraction. For the tissue distribution study, tissues and organs were collected from three mature female zebrafish. In addition, testis was sampled from three mature males. For the oogenesis study, stages I to IV ovarian follicles were dissected from ovaries collected from four mature zebrafish females according to developmental stages defined by (Selman et al., 1993). After removal from the body cavity, ovaries were subsequently incubated for $5 \mathrm{~min}$ in a Petri dish containing Hank's Full Strength solution (Westerfield, 2000) supplemented with Collagenase $(0.35$ $\mathrm{mg} / \mathrm{ml}$ ), then washed three times in large volume of Hank's Full Strength solution containing BSA $(0.5 \mathrm{mg} / \mathrm{ml})$. Ovarian follicles were then separated manually using forceps. Stage V oocytes were obtained from ovulating females by gentle manual stripping.

For the early development study, fertilized eggs were collected within 5-10 min of fertilization. For each batch, 15-20\% of the eggs were kept to assess developmental success and used to monitor survival at $24 \mathrm{hpf}$ and hatching rate. In order to analyze the developmental sequence of embryos originating from developmentally competent eggs, only egg batches exhibiting hatching rates above $80 \%$ were kept for further analysis. Remaining eggs of each batch were serially sampled at fertilization $(0.25 \mathrm{hpf}), 1$ cell $(0.75 \mathrm{hpf})$, sphere stage ( $4 \mathrm{hpf})$, shield stage (6 hpf), and $24 \mathrm{hpf}$, according to previously described developmental stages (Kimmel et al., 1995).

For in situ and whole mount in situ hybridization, ovaries or embryos were fixed overnight in $4 \%$ paraformaldehyde at $4{ }^{\circ} \mathrm{C}$ and then transferred in $100 \%$ methanol 
after three successive short incubations in methanol solution of increasing concentrations and subsequently conserved at $-20^{\circ} \mathrm{C}$ until further processing.

\section{PCR analysis}

For each sample, total RNA was extracted using Tri-Reagent (Molecular Research Center, Cincinnati, $\mathrm{OH}$ ) according to the manufacturer's instructions. Reverse transcription (RT) was performed using $2 \mu \mathrm{g}$ of RNA for each sample with MMLV reverse transcriptase and Random hexamers (Promega, Madison, WI). Briefly, RNA and dNTPs were denatured for $6 \mathrm{~min}$ at $70^{\circ} \mathrm{C}$, chilled on ice for 5 min before the reverse transcription master mix was added. Reverse transcription was performed at $37^{\circ} \mathrm{C}$ for $1 \mathrm{~h}$ and $15 \mathrm{~min}$ followed by a 15 -min incubation step at $70^{\circ} \mathrm{C}$. Control reactions were run without reverse transcriptase and used as negative control in the real-time PCR study. Quantitative RT-PCR (QPCR) experiments were performed using an Applied Biosystems StepOne Plus. RT products, including control reactions, were diluted to $1 / 25$, and $4 \mu 1$ were used for each PCR reaction. All QPCR reactions were performed in triplicates. QPCR was performed using a real-time PCR kit provided with a Fast-SYBR Green fluorophore (Applied Biosystems) with 100, 200, or $300 \mathrm{nM}$ of each primer in order to keep PCR efficiency between 90 and $100 \%$ for all target genes. In order to avoid unspecific signal due to genomic DNA contamination, primers (Table 1) were designed on exon boundaries whenever possible. The relative abundance of target cDNA within a sample set was calculated from serially diluted cDNA pool (standard curve) using Applied Biosystem StepOne ${ }^{\mathrm{TM}}$ V.2.0 software. After amplification, a fusion curve was obtained to validate the amplification of a single PCR product. The fusion curves obtained showed that each primer pair used was specific of a single nme transcript. The negative control reactions were used to estimate background level. Genes were considered significantly expressed when measured level was significantly above background at $p<0.05$ and within the range of the standard curve.

\section{Tissue specificity}

For each studied tissue, cDNA originating from three individual fish were pooled and subsequently used for real-time PCR. Before further analysis, real-time PCR data were collected using the same detection threshold for all studied genes. Data were subsequently normalized using the $\Delta \Delta \mathrm{Ct}$ method to $18 \mathrm{~S}$ transcript abundance in samples diluted to $1 / 2000$.

\section{Oogenesis and early development specificity}

For oogenesis and early development samples, 9 pg of exogenous luciferase RNA (Promega) was added per oocyte/embryo to the sample prior to homogenization in Tri-reagent. QPCR data were analyzed using the same detection threshold for all studied genes. Data were subsequently normalized using the $\Delta \Delta \mathrm{Ct}$ method to the exogenous luciferase transcript abundance in samples diluted to 1/25.

\section{In situ and whole-mount in situ hybridization}

Digoxigenin-labeled anti-sense RNA probes were produced using the Promega SP6/T7 RNA polymerase Riboprobe Combination System following manufacturer's instructions, using as DNA template a PCR product. Bacterial 
clones containing, nme2b1 (GenBank Acc\# FDR202-P00003-BR_F18), nme3 (GenBank Acc\# FDR103-P00026-BR_E16), nme4 (GenBank Acc\# FDR306P00036-BR_P09), and nme6 (GenBank Acc\# FDR202-P00041-BR_C14) inserts were obtained from the Genome Institute of Singapore. For nme3, nme4 and nme6 the PCR product used to generate the probe corresponds to the full-length clone. Due to high similarities between the three nme 2 transcripts, the $n m e 2 b 1$ anti-sense probe corresponds to the 3'UTR of the RNA sequence (nuc \# 478 to 624 of RefSeq NM_130926) that was generated using a specific forward primer (GCACAGCAGTGGATCTACGA) and M13 reverse primer. $n m e 2 b 1$ sense probe was synthesized on the full-length clone. For all plasmids, insert sequence was checked by sequencing. Digoxigenin-labeled riboprobes were then purified by precipitation in ammonium acetate $7.5 \mathrm{M}$ /ethanol for 2 hours at $-20^{\circ} \mathrm{C}$, and RNA concentrations were measured using a NanoDrop spectrophotometer. Samples dehydration (increasing ethanol: $15 \mathrm{~min}$ in 50\% ethanol, twice $15 \mathrm{~min}$ in $70 \%$ ethanol, $15 \mathrm{~min}$ in $80 \%$ ethanol, $30 \mathrm{~min}$ in $96 \%$ ethanol, and $30 \mathrm{~min}$ in $96 \%$ ethanol/butanolvol/vol), clearing (butanol once for $30 \mathrm{~min}$, and twice for $3 \mathrm{~h}$ each), and paraffin infiltration (once for $1 \mathrm{~h}$ and twice for $2 \mathrm{~h}$, at $60^{\circ} \mathrm{C}$ ) were performed in a Citadel 1000 tissue processor (Shandon, Pittsburgh, PA). Dehydrated tissues were embedded in paraffin using a HistoEmbedder (TBS88, Medite, Germany). For in situ hybridization (ISH), serial cross-sections of $5 \mu \mathrm{m}$ were deparaffinized, re-hydrated in TBS (50 mM Tris, $\mathrm{pH} 7.4,150 \mathrm{mM} \mathrm{NaCl}$ ) and post-fixed in 4\% PFA for $20 \mathrm{~min}$. ISH was performed using the In situ Pro, Intavis AG robotic station. Incubation volumes for all ISH steps were set to 250 $\mu \mathrm{l}$. Digestion was carried out for $20 \mathrm{~min}$ at $37^{\circ} \mathrm{C}$ with $2 \mu \mathrm{g} / \mathrm{ml}$ of proteinase $\mathrm{K}$. Pre-hybridization $\left(2 \mathrm{~h}, 60^{\circ} \mathrm{C}\right)$ and hybridization $\left(12 \mathrm{~h}, 60^{\circ} \mathrm{C}\right)$ were carried out in $50 \%$ formamide, 2 X SSC, 1 X Denhardt, $10 \%$ dextran sulfate, and $250 \mu \mathrm{g} / \mathrm{ml}$ tRNA. For hybridization, the digoxigenin-labeled anti-sense RNA probes were diluted in hybridization buffer at a final concentration of $8 \mathrm{ng} / \mu \mathrm{l}$. Washing steps $\left(6 \times 10 \mathrm{~min}, 60^{\circ} \mathrm{C}\right)$ were performed with $2 \times \mathrm{SSC}$ followed by an RNAse treatment at $37^{\circ} \mathrm{C}$. The digoxigenin signal was then revealed with an antidigoxigenin antibody conjugated with alkaline phosphatase (Roche Diagnostics Corp.) and a NBT/BCIP revelation system (Roche Diagnostics Corp.) as recommended by the manufacturer. Slides were mounted with mowiol 4-88 (Calbiochem).Whole-mount in situ hybridization (WISH) was also performed using the In situ Pro, Intavis AG robotic station. Incubation volumes for all WISH steps were set to $700 \mu \mathrm{l}$. Digestion was carried out for $20 \mathrm{~min}$ at $37^{\circ} \mathrm{C}$ with 25 $\mu \mathrm{g} / \mathrm{ml}$ of proteinase K. Samples were post-fixed in $4 \%$ PFA $/ 0.2 \%$ Glutaraldehyde for $40 \mathrm{~min}$ and then washed twice for $20 \mathrm{~min}$ in PBST. Embryo sample were not digested with proteinase $\mathrm{K}$. Pre-hybridization $\left(2 \times 1 \mathrm{~h}, 65^{\circ} \mathrm{C}\right)$ and hybridization $\left(16 \mathrm{~h}, 65^{\circ} \mathrm{C}\right)$ were carried out in $50 \%$ formamide, $5 \mathrm{X}$ SSC, $0.1 \%$ Tween 20 , $0.005 \%$ Heparine, and $100 \mu \mathrm{g} / \mathrm{ml}$ tRNA. For hybridization the digoxigeninlabeled anti-sense RNA probes were diluted in hybridization buffer at a final concentration of $2 \mathrm{ng} / \mu \mathrm{l}$. Post-hybridization washing steps $\left(3 \times 40 \mathrm{~min}, 65^{\circ} \mathrm{C}\right)$ were performed in 50\% Formamide / 2X SSC. Further washing steps (6 x 40 min, $55^{\circ} \mathrm{C}$ ) were performed in $2 \mathrm{X} \mathrm{SSC}$ and $0.2 \mathrm{X} \mathrm{SSC}$ and $(4 \times 40 \mathrm{~min}, \mathrm{RT})$ in PBST. The digoxigenin signal was then revealed with an anti-digoxigenin antibody conjugated with alkaline phosphatase (Roche Diagnostics Corp.) and a NBT/BCIP revelation system (Roche Diagnostics Corp.) as recommended by the manufacturer. For each studied gene, the duration of revelation was kept identical for all developmental stages assayed. Samples were then rinsed overnight in PBS and subsequently observed under binocular (Zeiss, Stemi 2000-C). 


\section{Statistical analysis}

Significant differences from negative sample and between samples were analyzed using Wilcoxon Rank Sum Test with superiority or inferiority alternatives hypothesis. All statistical analyses were performed using R v.2.6.2.

\section{Results}

\section{Tissue distribution of $n m e$ genes}

The tissue distribution study shows that most tissues exhibit very specific expression patterns of nme genes (Fig. 1). Among group I nme genes (nme2-4), $n m e 2 a, n m e 2 b 1$, and $n m e 2 b 2$ are the genes exhibiting the highest expression levels, while nme 3 and nme 4 are expressed at much lower levels. However, the copy of the $n m e 2$ gene exhibiting the highest expression is highly dependent on the tissue. For example, nme $2 a$ is the most highly expressed nme 2 gene in eyes and testis. In contrast, $n m e 2 b l$ is the most highly expressed $n m e 2$ gene in hepatopancreas, intestine and ovary while $n m e 2 b 2$ is the most highly expressed nme 2 gene in brain, muscle, and gills. For all these tissues with the exception of eyes and gills, there is a clear predominant expression of one nme 2 gene in comparison to the other nme 2 genes. In most of these tissues, the predominant nme 2 gene exhibits at least a 10-fold over expression in comparison to the two other $n m e 2$ genes. In contrast to group I, nme genes of the group II exhibit a very specific tissue distribution with the exception of nme6 that is expressed in most studied tissues. The ovary is the expression site exhibiting the highest level of nme 6 while nme 5 and nme 8 exhibit a testis-specific expression. In contrast to other tissues, the gonads exhibit a wide variety of nme gene expression with marked differences between male and female gonads. In the ovary, $n m e 2 b 1$, nme 3, nme 4 and nme 6 are the most expressed genes while nme 5 and nme 8 are the only ones that remain undetected. By contrast, nme5, nme $2 a$ and nme 8 are the most expressed nme genes in testis, while nme $2 b 1, n m e 3$ and nme 4 exhibit lower expression levels. In the testis, the expression of all nme genes could be detected above background levels.

\section{nme gene mRNA profiling during oogenesis}

The expression of nme genes exhibiting an ovarian expression was monitored in the ovary throughout the oogenetic process. Among all ovarian nme genes, only $n m e 2 b 1, n m e 3$, nme 4 and nme 6 were significantly expressed in oocytes and ovarian follicles. In contrast, the expression levels of nme $2 a, n m e 2 b 2$ and nme 7 were extremely low in ovarian tissue (Fig. 1), ovarian follicles and oocytes (data not shown). Among studied genes, $n m e 2 b 1$ is the most expressed gene with expression levels 9,11 and 18 times higher than nme6, nme 3 and nme4, respectively. As shown by the in situ analysis, the expression of nme2b1, nme3, nme 4 and nme6 was shown to be restricted to the oocyte, as no signal was observed in follicular layers (Fig.2). These 4 genes exhibited a similar expression profile during oogenesis (Fig. 2). The highest expression levels per oocyte were observed in stage I oocytes. The levels of transcript subsequently decreased throughout oogenesis. A drop of mRNA levels of nme $2 b 1$, nme 3 and nme 4 was observed in stage II oocytes in comparison to stage I, while this drop was not statistically significant for nme6. The mRNA levels of nme4 subsequently 
exhibited a step-wise decrease from stage III to stage V of oocyte development. A decrease in $n m e 2 b 1$, nme 3 and nme6 mRNA levels was further observed in stage IV and stage V oocytes in comparison to stage III.

\section{nme gene mRNA profiling during early development}

During early development, only nme $2 b 1, n m e 2 b 2, n m e 3$ and nme6 were found to be significantly expressed in the zebrafish embryo (Fig. 3). In contrast, nme $2 a$, $n m e 4, n m e 5$, and nme 7 were either not detected or expressed at very low levels (data not shown). Significant levels of nme 3 and nme6 transcripts were detected in fertilized zebrafish eggs, thus demonstrating their maternal origin. In contrast, $n m e 2 b 1$ and $n m e 2 b 2$ were not significantly detected in fertilized eggs. The transcripts of nme $2 b 1, n m e 2 b 2$ and nme 3 were weakly detected before MBT but were actively transcribed after zygotic genome activation as shown by their high expression level at $24 \mathrm{hpf}$. In contrast, nme6 displayed a different pattern as corresponding mRNA levels decreased very quickly after fertilization. Similarly to $n m e 2 b 1, n m e 2 b 2$, and $n m e 3, n m e 6$ was actively expressed after MBT. When comparing expression levels of those 4 genes, nme $2 b 2$ was the most expressed gene at $24 \mathrm{hpf}$.

\section{Early development whole-mount in situ hybridization}

As indicated above, nme 3 and nme6 are the only nme mRNAs maternally inherited by the zebrafish embryo. Using WISH we observed that nme 3 transcript labeling can be detected in the zebrafish embryo at 1cell and $4 \mathrm{hpf}$ but not at $6 \mathrm{hpf}$ (Fig. 4B). At $24 \mathrm{hpf}$, nme 3 transcript was localized in the eyes, the brain with a strong signal in midbrain and hindbrain. nme 3 transcript was also detected all along the neural tube with a stronger labeling in the tail bud region (Fig. 4B). Weak nme3 signal could also be observed along the pronephric duct. nme6 transcript labeling was moderate and ubiquitous from 1cell stage to $6 \mathrm{hpf}$ (Fig. 4B). At $24 \mathrm{hpf}$, a moderate labeling was observed in the eyes and the brain, and a weak signal was present in the somites (Fig. 4B).

\section{Discussion}

\section{Zebrafish nme gene expression patterns}

In mammals, the Nme gene family has been extensively studied, mostly because of its role in metastatic process (Boissan et al., 2009). The amount of available information, is however, very different depending on the member of the family. While specific Nme family members such as Nme1 and Nme 2 have been thoroughly studied, other Nme genes (e.g. Nme6) have received far less attention. In addition, the analysis of existing data is further complicated by the numerous lineage specific gene duplications of group I Nme genes that have been found in all metazoans, including mammals and teleost (Desvignes et al., 2009 ; Desvignes et al., 2010). For instance, mammalian Nme1, Nme2, Nme3, and Nme4 genes are co-orthologs of $A w d$, the only group I Nme gene found in the Drosophila genome (Desvignes et al., 2010). Similarly, mammalian Nmel and Nme2 genes are coorthologs of zebrafish nme $2 a, n m e 2 b 1$, and nme2b2. In contrast, orthology relationships of Nme 3 and Nme 4 genes were clearly established among vertebrates, including zebrafish and mammals (Desvignes et al., 2009). Similarly, 
a single ortholog of each group II Nme gene (Nme5, Nme6, Nme7, and Nme8) could be found in all metazoan species with some exceptions in species in which some genes highly diverged or were lost (Desvignes et al., 2010). Together, these observations suggest that the expression patterns and tissue distribution of group II Nme genes can be compared among Metazoans. Similarly, the expression patterns and tissue distribution of Nme 3 and Nme 4 can be compared among vertebrates. In contrast, interpretation of expression data regarding Nme1/Nme 2 genes among vertebrates and metazoans is much more difficult due to independent lineage-specific duplications.

In mouse, Nme2 is widely and ubiquitously expressed among tissues and organs whereas Nmel expression can be highly variable depending on tissues, despite a broadly distributed expression (Barraud et al., 2002). Within a specific mammalian tissue, the Nme1/Nme2 expression ratio is however highly variable (Tsuchiya et al., 1998 ; Barraud et al., 2002). Together, these observations are consistent with the widely distributed expression of $n m e 2$ genes reported here in zebrafish. It has been shown that all three $n m e 2$ zebrafish paralogous genes originate from a unique vertebrate nme 2 ancestral gene. Similarly, mammalian Nmel and Nme2 originate from the same unique nme 2 ancestral gene (Desvignes et al., 2009). It is thus tempting to postulate that ancestral nme 2 functions could have been ubiquitous and multifunctional among tissues, and that $N m e 2$ genes may have undergone subsequent sub-functionalization, at least in some organs, after successive gene duplication events (Zhang, 2003). For example, it has been shown that Nme1 is preferentially expressed in the nervous system of tetrapods and more specifically in the brain (Kimura et al., 1990 ; Ouatas et al., 1998 ; Dabernat et al., 1999a ; Dabernat et al., 1999b ; Barraud et al., 2002). This suggests that Nme1 may have retained or developed a specific function in the nervous system that $N m e 2$ may not have. In zebrafish, we show that $n m e 2 b 2$ is the most expressed gene in the brain, thus also suggesting that following duplication events in teleosts, $n m e 2 b 2$ has also retained this cerebral function in contrast to $n m e 2 a$ and $n m e 2 b 1$ that are poorly expressed in the brain. In this context, we can therefore speculate that the $n m e 2$ ancestor gene has undergone multiple functional changes in vertebrates following the successive duplication events. Among the very specific tissue distribution of the zebrafish nme 2 genes, the very high expression of $n m e 2 b 2$ in muscle should be noted. In addition to being expressed in all assayed tissues at moderate levels, $n m e 2 b 2$ exhibits a highly predominant expression in muscular tissue that has never been described in any other vertebrate species. This expression pattern is in striking contrast with existing Northern blot data reporting a low or moderate expression of Nme1 and Nme2 in mammalian skeletal muscle (Tsuchiya et al., 1998 ; Dabernat et al., 1999b ; Masse et al., 2002). Similarly to $n m e 2 b 2, n m e 2 b 1$ is also widely distributed with a predominant expression in the ovary. In contrast to nme 2 genes, other $n m e$ genes (i.e. nme3-8) display more specific tissue distributions that are also observed in other vertebrate species. For instance, nme 5 and nme 8 exhibit a strict testis specific expression in agreement with existing data in mammals (Munier et al., 1998 ; Sadek et al., 2001 ; Hwang et al., 2003 ; Miranda-Vizuete et al., 2003). Similarly, nme3 and nme6 are widely distributed in zebrafish tissues and organs in agreement with existing data in mammals (Mehus et al., 1999 ; Masse et al., 2002). However, the predominant ovarian expression of nme 3 and nme6 has never been documented in mammals, birds or amphibians. Finally, it is noteworthy that in comparison to other studied tissues, reproductive organs express a wide variety of $n m e$ genes. 
While nme 4 and nme 7 are both expressed at comparable levels in ovary and testis, $n m e 2 b 1, n m e 3$, and nme 6 are expressed at higher levels in the ovary, whereas nme $2 a, n m e 5$, and nme 8 are strongly expressed in the testis.

\section{Nme genes in zebrafish oocyte and early embryo development}

\section{Nme gene expression during oogenesis}

During zebrafish oogenesis, nme $2 b 1, n m e 3, n m e 4$ and nme6 are expressed at significant levels in the oocyte. These observations are consistent with the tissue distribution study reported here. Similarly, the lack of nme $2 a, n m e 2 b 2$, and nme 7 expression is also consistent with low ovarian expression level reported in (Desvignes et al., 2009) and the present study. It is noteworthy that all ovarian nme genes display a decreasing expression profile during oogenesis, the decrease in oocyte expression levels being more progressive for $n m e 6$ and $n m e 3$ in comparison to $n m e 2 b 1$ and $n m e 4$. This suggests that $n m e 2 b 1$ and nme4 are translated during oogenesis. It is thus possible that the corresponding proteins are present in the mature oocyte and are subsequently maternally-inherited. The strong decrease in mRNA levels in the oocyte between stage III and stage V would be in favor of this hypothesis. This would also be consistent with the identification of an Nme2b-related protein in zebrafish and gilthead seabream (Sparus aurata) oocytes during maturation (Ziv et al., 2008), an Nme2-related protein identified in Persian sturgeon (Acipenser persicus) mature oocytes (Keyvanshokooh and Vaziri, 2008), and an Nme2-related protein in sea bass (Dicentrarchus labrax) eggs (Crespel et al., 2008). Using ISH, we confirmed that the transcripts are expressed in the oocyte and not in surrounding follicular layers. The labeling is predominant in small oocytes and not-discernable in stage IV oocytes. The absence of labeling in the last stages of oogenesis can be explained by a dilution effect due to the dramatic increase in oocyte volume due to major yolk accumulation (Lubzens et al., 2010). In addition, QPCR data clearly demonstrated that full-grown oocytes contain nme2b1, nme3, nme4, and nme6 transcripts. Together, our observations indicate that nme2b1, nme3, nme4, and nme6 mRNAs are maternally-inherited.

\section{Nme gene expression during early development}

During zebrafish early development, only nme $2 b 1, n m e 2 b 2$, nme3, and nme6 could be detected at significant levels. At pre-MBT stages, only nme3 and nme6 could be detected, in agreement with expression levels in stage $\mathrm{V}$ oocytes (see above). In contrast, the lack of detection of nme 4 and nme $2 b 1$ transcripts in fertilized eggs suggests a drop of transcript abundance either during postovulatory ageing (Aegerter et al., 2005) and/or during fertilization and activation, before the formation of the first embryonic cell. The lack of nme $2 a$ expression is consistent with previous reports of predominant expression in eyes and testis (Desvignes et al., 2009), organs that are not fully developed at $24 \mathrm{hpf}$. In addition, $n m e 2 b 1$ and $n m e 2 b 2$ display low levels of expression before $24 \mathrm{hpf}$ in comparison to $n m e 3$ and nme 6 that are detected from fertilization to $6 \mathrm{hpf}$. Because of this very low expression level of $n m e 2 b 1$ and $n m e 2 b 2$ before $24 \mathrm{hpf}$, these genes were not studied by whole mount in situ hybridization. This observation is consistent with the report of an $n m e 2 b$-related transcript in Atlantic halibut during embryonic development (Hippoglossus hippoglossus) (Bai et al., 2007). The very 
high expression of $n m e 2 b 1$ at $24 \mathrm{hpf}$, a period when organogenesis occurs, is consistent with the wide distribution of nme $2 b 1$ transcript in zebrafish tissues (Desvignes et al., 2009). This observation is also consistent with the observations made in Atlantic salmon (Salmo salar) embryos in which an nme 2 transcript corresponding to an $n m e 2 b$ was cloned and detected only after gastrulation (Murphy et al., 2000). Similarly, this is also consistent with prior report of the knock-down of $n m e 2 b 1$ and $n m e 2 b 2$ expression in zebrafish embryos that did not induce any apparent phenotype before $24 \mathrm{hpf}$ (Hippe et al., 2009). Furthermore, in Xenopus laevis, no nme2-related transcript could be detected before MBT (Ouatas et al., 1998). In the 24 hpf embryo, nme $2 b 2$ is the most abundant $n m e$ transcript in agreement with the high expression level observed in adult muscle.

\section{nme3 in zebrafish development}

As described above, nme 3 displays a typical maternal RNA profile as it is expressed in the oocytes during oogenesis and present in the embryo during early development. During the first hours of development, abundance levels are stable. Using WISH, we confirmed that nme3 transcript was present in the embryo from 1 cell stage to $4 \mathrm{hpf}$. At $6 \mathrm{hpf}$, a lack of signal can be observed that may result from a dilution effect of the RNA within the embryo. At $24 \mathrm{hpf}$, a weak expression signal can be observed along the pronephric duct. The expression of nme3 in fish kidney was, however previously unreported and would require further analysis. Interestingly, nme 3 transcript labeling is predominant along the nervous systems as revealed by a strong signal in brain and eyes and a moderate signal all along the neural tube. This localization is in total agreement with expression patterns of nme 3 observed in adult mouse tissues and during mouse organogenesis with highest expression in nervous system (Masse et al., 2002 ; Amrein et al., 2005). Such a similar expression pattern between mouse and zebrafish strongly suggest that nme 3 could be an important factor common to all vertebrates for nervous and sensory system development.

\section{nme6 in zebrafish development}

As indicated above, nme 6 also display a typical maternal mRNA profile during zebrafish early development. However, in contrast to nme3 that is stable during the first 6 hour post-fertilization, we show here using both QPCR and WISH that nme6 abundance displays a rapid decrease during the first cell cycles. This decrease of nme 6 transcript abundance suggests an important translational activity and an important role in the very first steps of development. At $24 \mathrm{hpf}$, nme6 is actively transcribed and its expression is mainly localized in the brain, the eyes and in the somites. This localization is consistent with expression data of nme6 among zebrafish tissues.

\section{Conclusion}

The present study provides new insights into the fish nme gene family by providing a complete expression survey of the entire family during zebrafish oogenesis and early development. We show that nme2b1, nme3, nme4, and nme6 are highly expressed in the ovary and present in the oocyte throughout oogenesis. While the abundance of the four transcripts exhibit a continuous decrease throughout oogenesis, nme 3 and nme6, clearly display a typical maternal mRNA 
Abrams EW, Mullins MC (2009) Early zebrafish development: It's in the maternal genes. Current Opinion in Genetics \& Development 19:396-403

Aegerter S, Jalabert B, Bobe J (2005) Large scale real-time PCR analysis of mRNA abundance in rainbow trout eggs in relationship with egg quality and post-ovulatory ageing. Mol Reprod Dev $72: 377-385$

Amrein L, Barraud P, Daniel JY, Perel Y, Landry M (2005) Expression patterns of nm23 genes during mouse organogenesis. Cell Tissue Res 322:365-378

Bai JL, Solberg C, Fernandes JMO, Johnston IA (2007) Profiling of maternal and developmentalstage specific mRNA transcripts in Atlantic halibut Hippoglossus hippoglossus. Gene 386:202-210

Barraud P, Amrein L, Dobremez E, Dabernat S, Masse K, Larou M, Daniel JY, Landry M (2002) Differential expression of nm23 genes in adult mouse dorsal root ganglia. J Comp Neurol 444:306-323

Biggs J, Hersperger E, Steeg PS, Liotta LA, Shearn A (1990) A Drosophila gene that is homologous to a mammalian gene associated with tumor metastasis codes for a nucleoside diphosphate kinase. Cell 63:933-940

Bilitou A, Watson J, Gartner A, Ohnuma SI (2009) The NM23 family in development. Mol Cell Biochem

Bobe J, Labbe C (2010) Egg and sperm quality in fish. Gen Comp Endocrinol 165:535-548

Boissan M, Dabernat S, Peuchant E, Schlattner U, Lascu I, Lacombe ML (2009) The mammalian Nm23/NDPK family: from metastasis control to cilia movement. Mol Cell Biochem 329:51-62

Carotenuto P, Marino N, Bello AM, D'Angelo A, Di Porzio U, Lombardi D, Zollo M (2006) PRUNE and NM23-M1 expression in embryonic and adult mouse brain. J Bioenerg Biomembr $38: 233-246$

Crespel A, Rime H, Fraboulet E, Bobe J, Fauvel C (2008) Egg quality in domesticated and wild seabass (Dicentrarchus labrax): A proteomic analysis. Cybium 32(2) suppl.:205

Dabernat S, Larou M, Masse K, Hokfelt T, Mayer G, Daniel JY, Landry M (1999a) Cloning of a second nm23-M1 cDNA: expression in the central nervous system of adult mouse and comparison with nm23-M2 mRNA distribution. Brain Res Mol Brain Res 63:351-365 
Dabernat S, Larou M, Masse K, Dobremez E, Landry M, Mathieu C, Daniel JY (1999b)

Organization and expression of mouse nm23-M1 gene. Comparison with nm23-M2 expression. Gene 236:221-230

Desvignes T, Pontarotti P, Bobe J (2010) Nme Gene Family Evolutionary History Reveals PreMetazoan Origins and High Conservation between Humans and the Sea Anemone, Nematostella vectensis. PLoS ONE 5:e15506

Desvignes T, Pontarotti P, Fauvel C, Bobe J (2009) Nme protein family evolutionary history, a vertebrate perspective. BMC Evolutionary Biology 9:256

Dosch R, Wagner DS, Mintzer KA, Runke G, Wiemelt AP, Mullins MC (2004) Maternal Control of Vertebrate Development before the Midblastula Transition: Mutants from the Zebrafish I. Developmental Cell 6:771-780

Hippe HJ, Wolf NM, Abu-Taha I, Mehringer R, Just S, Lutz S, Niroomand F, Postel EH, Katus HA, Rottbauer W, Wieland T (2009) The interaction of nucleoside diphosphate kinase B with $\mathrm{G}+,+i$ dimers controls heterotrimeric $\mathrm{G}$ protein function. Proceedings of the National Academy of Sciences 106:16269-16274

Hwang KC, Ok DW, Hong JC, Kim MO, Kim JH (2003) Cloning, sequencing, and characterization of the murine nm23-M5 gene during mouse spermatogenesis and spermiogenesis. Biochemical and Biophysical Research Communications 306:198-207

Kane DA, Kimmel CB (1993) The zebrafish midblastula transition. Development 119:447-456

Keyvanshokooh S, Vaziri B (2008) Proteome analysis of Persian sturgeon (Acipenser persicus) ova. Animal Reproduction Science 109:287-297

Kimmel CB, Ballard WW, Kimmel SR, Ullmann B, Schilling TF (1995) Stages of embryonic development of the zebrafish. Dev Dyn 203:253-310

Kimura N, Shimada N, Nomura K, Watanabe K (1990) Isolation and characterization of a cDNA clone encoding rat nucleoside diphosphate kinase. J Biol Chem 265:15744-15749

Lakso M, Steeg PS, Westphal H (1992) Embryonic Expression of Nm23 During Mouse Organogenesis. Cell Growth \& Differentiation 3:873-879

Lubzens E, Young G, Bobe J, Cerda J (2010) Oogenesis in teleosts: how eggs are formed. Gen Comp Endocrinol 165:367-389

Masse K, Dabernat S, Bourbon PM, Larou M, Amrein L, Barraud P, Perel Y, Camara M, Landry M, Lacombe ML, Daniel JY (2002) Characterization of the nm23-M2, nm23-M3 and nm23-M4 mouse genes: comparison with their human orthologs. Gene 296:87-97

Mathavan S, Lee SG, Mak A, Miller LD, Murthy KR, Govindarajan KR, Tong Y, Wu YL, Lam SH, Yang H, Ruan Y, Korzh V, Gong Z, Liu ET, Lufkin T (2005) Transcriptome analysis of zebrafish embryogenesis using microarrays. PLoS Genet 1:260-276

Mehus JG, Deloukas P, Lambeth DO (1999) NME6: a new member of the nm23/nucleoside diphosphate kinase gene family located on human chromosome 3p21.3. Human Genetics 104:454459

Miranda-Vizuete A, Tsang K, Yu Y, Jimenez A, Pelto-Huikko M, Flickinger CJ, Sutovsky P, Oko R (2003) Cloning and Developmental Analysis of Murid Spermatid-specific Thioredoxin-2 (SPTRX-2), a Novel Sperm Fibrous Sheath Protein and Autoantigen. J Biol Chem 278:4487444885 
Munier A, Feral C, Milon L, Pinon VPB, Gyapay G, Capeau J, Guellaen G, Lacombe ML (1998) A new human nm23 homologue (nm23-H5) specifically expressed in testis germinal cells. Febs Letters 434:289-294

Murphy M, Harte T, McInerney J, Smith TJ (2000) Molecular cloning of an Atlantic salmon nucleoside diphosphate kinase cDNA and its pattern of expression during embryogenesis. Gene 257:139-148

Ouatas T, Selo M, Sadji Z, Hourdry J, Denis H, Mazabraud A (1998) Differential expression of nucleoside diphosphate kinases (NDPK/NM23) during Xenopus early development. International Journal of Developmental Biology 42:43-52

Pelegri F (2003) Maternal factors in zebrafish development. Dev Dyn 228:535-554

Postel EH, Zou X, Notterman DA, La Perle KM (2009) Double knockout Nme1/Nme2 mouse model suggests a critical role for NDP kinases in erythroid development. Mol Cell Biochem $329: 45-50$

Sadek CM, Damdimopoulos AE, Pelto-Huikko M, Gustafsson JA, Spyrou G, Miranda-Vizuete A (2001) Sptrx-2, a fusion protein composed of one thioredoxin and three tandemly repeated NDPkinase domains is expressed in human testis germ cells. Genes Cells 6:1077-1090

Selman K, Wallace RA, Sarka A, Qi X (1993) Stages of Oocyte Development in the Zebrafish, Brachydanio rerio. Journal of Morphology 218:203-224

Tadros W, Lipshitz HD (2009) The maternal-to-zygotic transition: a play in two acts. Development 136:3033-3042

Timmons L, Shearn A (2000) Role of AWD/nucleoside diphosphate kinase in Drosophila development. J Bioenerg Biomembr 32:293-300

Tsuchiya B, Sato Y, Urano T, Baba H, Shiku H, Kameya T (1998) Immunohistochemical and Semiquantitative Immunoblot Analyses of Nm23-H1 and H2 Isoforms in Normal Human Tissues. Acta Histochem Cytochem 31:411-418

Wagner DS, Dosch R, Mintzer KA, Wiemelt AP, Mullins MC (2004) Maternal Control of Development at the Midblastula Transition and beyond: Mutants from the Zebrafish II. Developmental Cell 6:781-790

Westerfield M (2000) The zebrafish book. A guide for the laboratory use of zebrafish (Danio rerio). Univ.of Oregon Press, Eugene

Woolworth JA, Nallamothu G, Hsu T (2009) The Drosophila Metastasis Suppressor Gene Nm23 Homolog, awd, Regulates Epithelial Integrity during Oogenesis. Mol Cell Biol 29:4679-4690

Zhang J (2003) Evolution by gene duplication: an update. Trends in Ecology \& Evolution 18:292298

Ziv T, Gattegno T, Chapovetsky V, Wolf H, Barnea E, Lubzens E, Admon A (2008) Comparative proteomics of the developing fish (zebrafish and gilthead seabream) oocytes. Comparative Biochemistry and Physiology Part D: Genomics and Proteomics 3:12-35 


\section{Tables}

Table1. QPCR primer sequences and accession numbers of target genes.

\begin{tabular}{cccc}
\hline $\begin{array}{c}\text { Target } \\
\text { gene }\end{array}$ & GenBankAcc\# & Forward sequence & Reverse sequence \\
\hline 18S & XM_001922869 & CGGAGGTTCGAAGACGATCA & GAGGTTTCCCGTGTTGAGTC \\
$n m e 2 a$ & NM_199970 & GACTGCCATCAAACAATTCCAA & AAGATCCTCATCTGCCTGGATTA \\
$n m e 2 b 1$ & NM_130926 & CCAGACGGGTTCTGTTCTGT & TGCTTGAGAAAAGACGAGATGA \\
$n m e 2 b 2$ & NM_130927 & TGGAGCTCAGAGTCCCTGTT & GGGTTCTGCTGTGTGTGTGT \\
$n m e 3$ & NM_130928 & TCCTGCACGGAGAAGATGAT & AACTCCATCCGGCTTCACT \\
$n m e 4$ & NM_201195 & TCAGCTGTTGGGTGGCATT & TAACCCTCGGACCGTTACAC \\
$n m e 5$ & NM_001002516 & GACGCATCCTGACTGTCTGA & ATGGGGAAACATGAACCTGA \\
$n m e 6$ & NM_131597 & GCACACATGAGGATCACTGG & CATCAAGTGCAGCAGAAGGA \\
$n m e 7$ & NM_130929 & GGTTGTGCCGAGTACCCTA & CATCTGGGGCATGTATTCC \\
$n m e 8$ & NM_001089475 & ATCAAGAACGAGCTGGGAGA & TCCCGCATAAAACAGAAAGG \\
\hline
\end{tabular}

\section{Legends}

Fig.1 Zebrafish tissue nme gene expression profile. nme gene expression in zebrafish eyes, brain, hepatopancreas, intestine, muscle, gills, ovary and testis. Mean and SD are shown $(n=4)$. \#, not detected or detected at extremely low levels. For all tissues, gene expression level is normalized by $18 \mathrm{~S}$ gene expression using the $\Delta \Delta \mathrm{Ct}$ method so that gene abundance can be compared among tissues.

Fig.2 Zebrafish nme2b1, nme3, nme4 and nme6 gene expression during oogenesis. Oocyte developmental stages according to Selman et al (1993). Mean and SD are shown ( $\mathrm{n}=4$ for all stages I, II, III, and V, $\mathrm{n}=5$ for stage IV). Different letters indicate significantly different levels of expression at $p<0.05$. For all stages, gene expression level is normalized by exogenous luciferase RNA abundance using the $\Delta \Delta \mathrm{Ct}$ method so that gene abundance can be compared among stages. Expression level was arbitrarily set to 1 for the lowest value of the data set (nme4 stage V). For each gene, an ISH picture of an ovary section is displayed to demonstrate the expression of the corresponding nme gene in the oocytes and the lack of expression in follicular layers. fl, follicular layers; $\mathrm{n}$, nucleus ; o, oocytes, the oocyte developmental stage is shown in brackets.

Fig.3 Zebrafish nme2b1, nme2b2, nme3 and nme6 gene expression during early development. Embryonic developmental stages according to Kimmel et al (1995). Mean and SD are shown ( $=4$ for zyg, $4 \mathrm{hph}, 6 \mathrm{hpf}$ and $24 \mathrm{hpf}, \mathrm{n}=3$ for 1 cell stage). Different letters indicate significantly different levels of expression at $\mathrm{p}<0.05$. For all stages, gene expression level is normalized by exogenous luciferase RNA expression using the $\Delta \Delta \mathrm{Ct}$ method so that gene abundance can be compared among stages. Expression level was arbitrarily set to 1 for the lowest value of the data set (nme2b1, Zyg). Zyg, correspond to the fertilized egg, before the formation of the first embryonic cell.

Fig.4 In situ hybridization and whole-mount in situ hybridization of nme3 and nme 6 transcripts during zebrafish oogenesis and early development. A. Corresponding zone hybridized with sense probe is shown in smaller size. I, stage I oocytes; II, stage II oocytes; IIa, stage IIa oocytes; IIb, stage IIb oocytes; III, 
stage III oocytes; IV, stage IV oocytes. B. Early development localization of nme3 and nme6 transcripts examined by whole-mount in situ hybridization. b, brain; hb, hindbrain; mb, midbrain; nt, neural tube; pd, pronephric duct; s, somites ; tb, tail bud; e, eyes. 

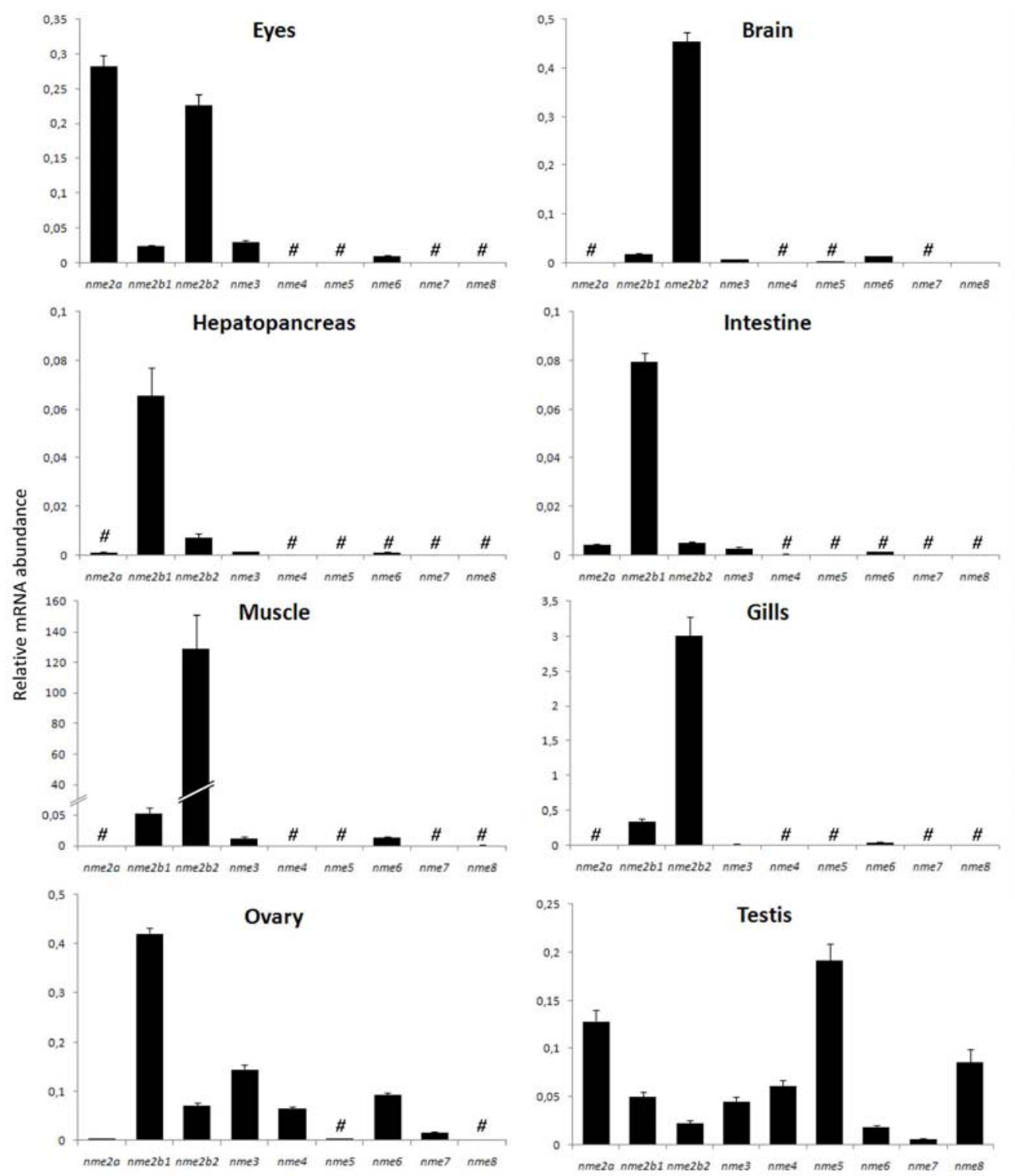

Zebrafish tissue nme gene expression profile. nme gene expression in zebrafish eyes, brain, hepatopancreas, intestine, muscle, gills, ovary and testis. Mean and SD are shown $(n=4)$. \#, not detected or detected at extremely low levels. For all tissues, gene expression level is normalized by $18 \mathrm{~S}$ gene expression using the $\Delta \Delta \mathrm{Ct}$ method so that gene abundance can be compared among tissues $173 \times 205 \mathrm{~mm}(600 \times 600 \mathrm{DPI})$ 


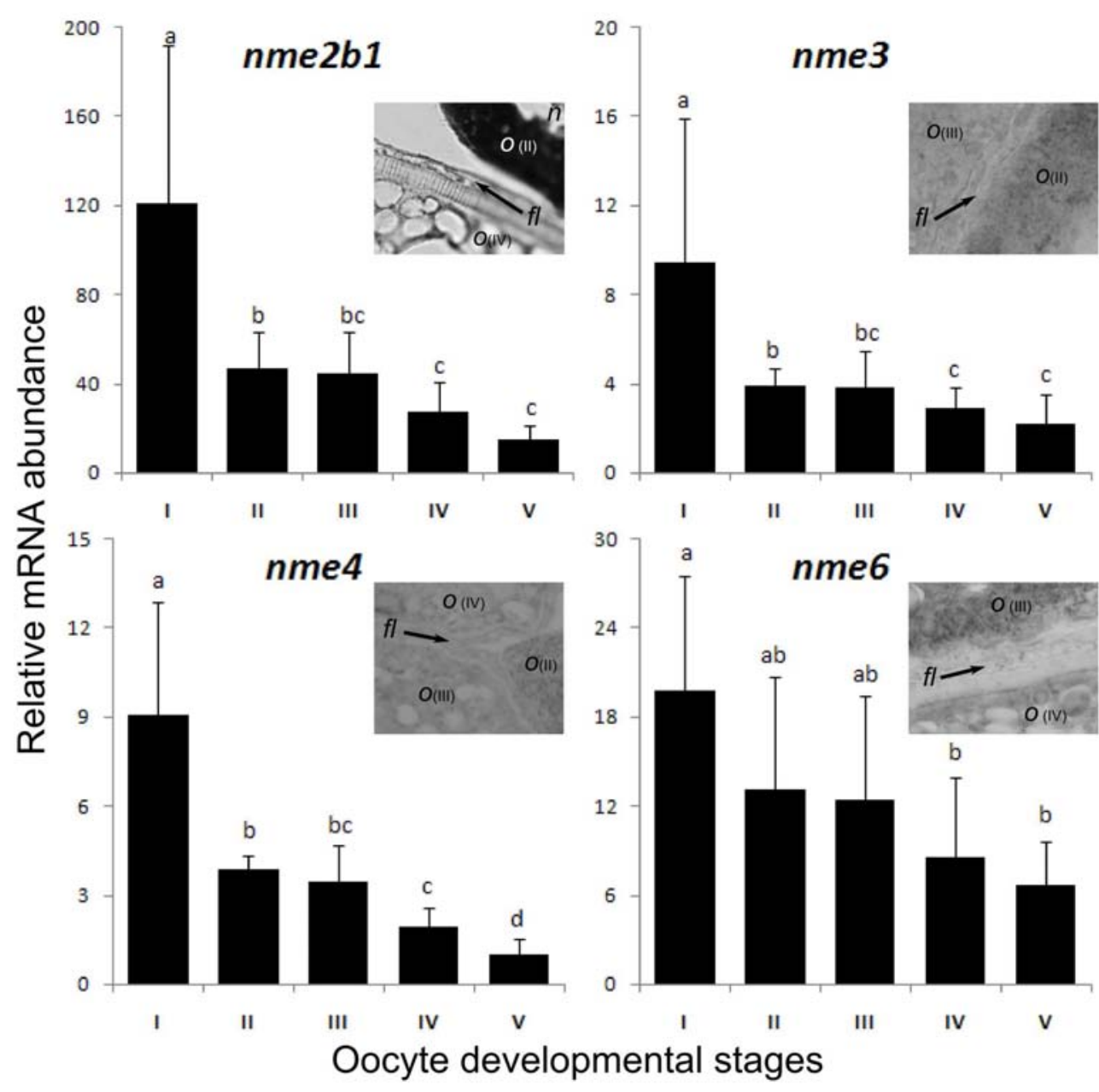

Fig. 2 Zebrafish nme2b1, nme3, nme4 and nme6 gene expression during oogenesis. Oocyte developmental stages according to Selman et al (1993). Mean and SD are shown ( $\mathrm{n}=4$ for all stages I, II, III, and V, $n=5$ for stage IV). Different letters indicate significantly different levels of expression at $p<0.05$. For all stages, gene expression level is normalized by exogenous luciferase RNA abundance using the $\Delta \Delta$ Ct method so that gene abundance can be compared among stages. Expression level was arbitrarily set to 1 for the lowest value of the data set (nme 4 stage $\mathrm{V}$ ). For each gene, an ISH picture of an ovary section is displayed to demonstrate the expression of the corresponding nme gene in the oocytes and the lack of expression in follicular layers. fl, follicular layers ; n, nucleus ; o, oocytes, the oocyte developmental stage is shown in brackets. $83 \times 81 \mathrm{~mm}(600 \times 600 \mathrm{DPI})$ 


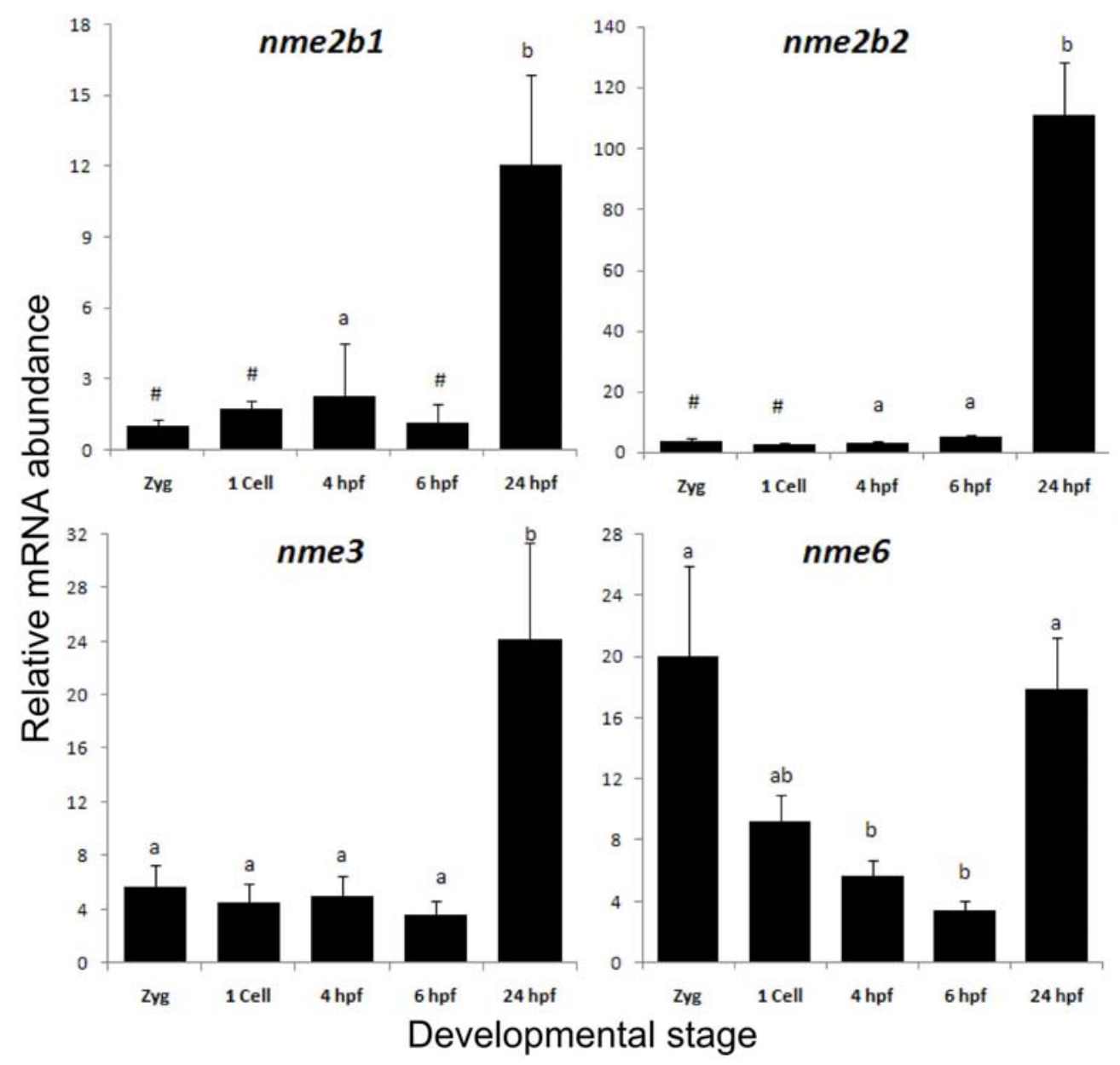

Fig.3 Zebrafish nme2b1, nme2b2, nme3 and nme6 gene expression during early development. Embryonic developmental stages according to Kimmel et al (1995). Mean and SD are shown $(n=4$ for zyg, $4 \mathrm{hph}, 6 \mathrm{hpf}$ and $24 \mathrm{hpf}, \mathrm{n}=3$ for 1 -cell stage). Different letters indicate significantly different levels of expression at $p<0.05$. For all stages, gene expression level is normalized by exogenous luciferase RNA expression using the $\Delta \Delta \mathrm{Ct}$ method so that gene abundance can be compared among stages. Expression level was arbitrarily set to 1 for the lowest value of the data set (nme2b1, Zyg). $\mathrm{Zyg}$, correspond to the fertilized egg, before the formation of the first embryonic cell. $83 \times 80 \mathrm{~mm}(600 \times 600 \mathrm{DPI})$ 
a
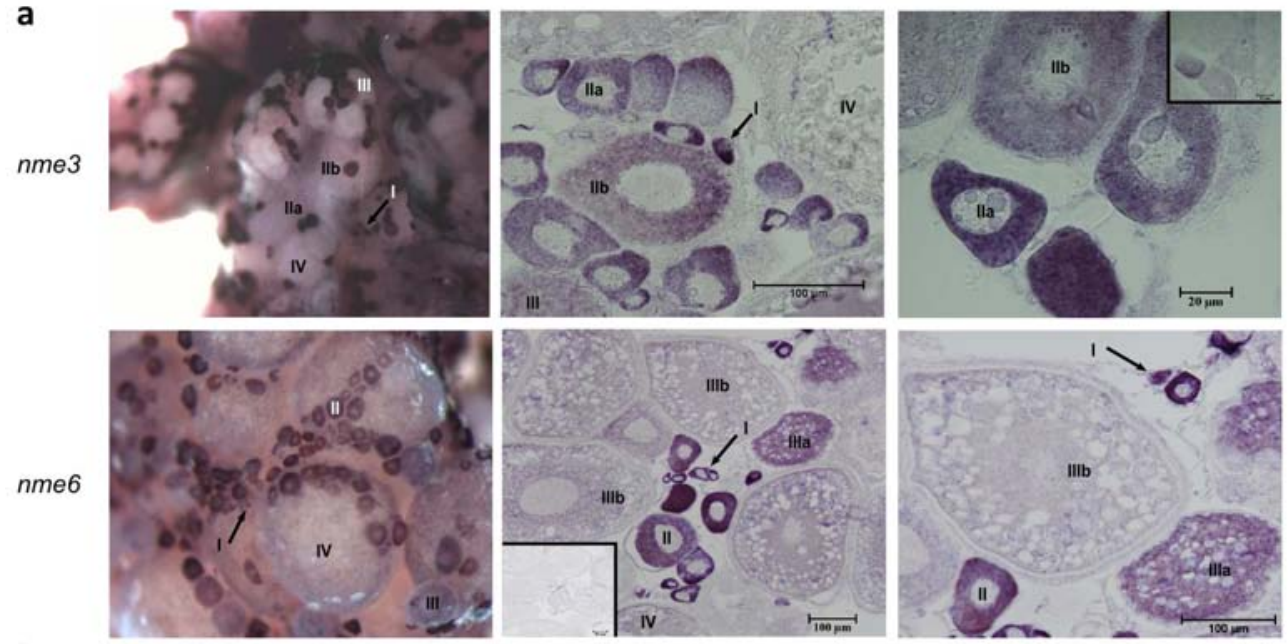

b

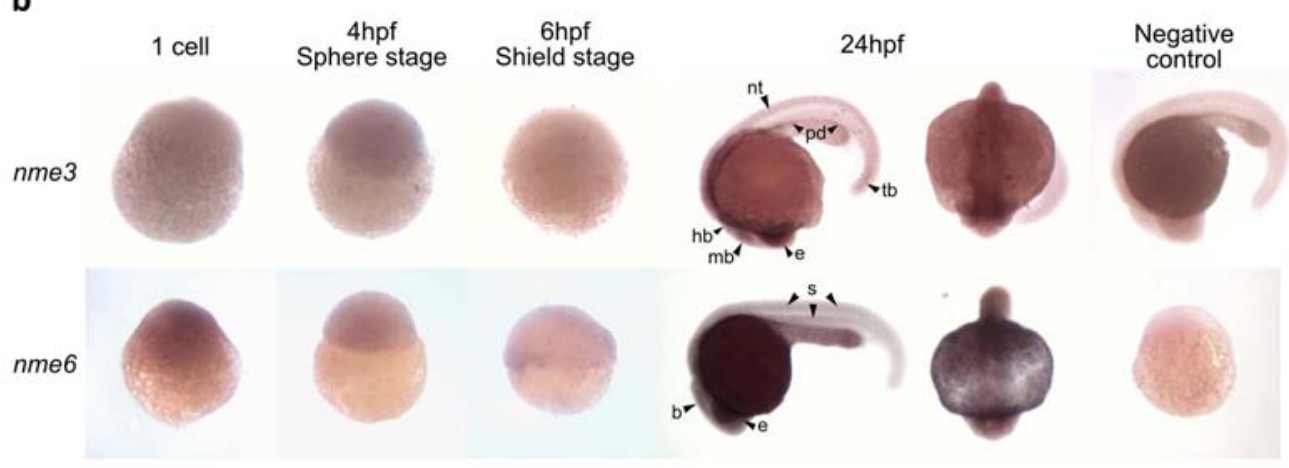

In situ hybridization and whole-mount in situ hybridization of nme3 and nme 6 transcripts during zebrafish oogenesis and early development. A. Corresponding zone hybridized with sense probe is shown in smaller size. I, stage I oocytes; II, stage II oocytes; IIa, stage IIa oocytes; IIb, stage IIb oocytes; III, stage III oocytes; IV, stage IV oocytes. B. Early development localization of nme3 and nme6 transcripts examined by whole-mount in situ hybridization. b, brain; hb, hindbrain; mb, midbrain; nt, neural tube; pd, pronephric duct; s, somites ; tb, tail bud; e, eyes. $173 \times 151 \mathrm{~mm}(300 \times 300 \mathrm{DPI})$ 